\title{
Cumulative cortisol exposure in the third trimester correlates with postpartum mothers' neural re- sponse to emotional interference
}

\author{
S.Stickel ${ }^{1,5}$, S. Eickhoff ${ }^{2,3}$, T. W. Goecke ${ }^{4}$, F. Schneider ${ }^{1,6}$, N. Soares Quinete ${ }^{7,8}$, J. Lang ${ }^{7}$, U. Habel ${ }^{1,5}$, N. \\ Chechko ${ }^{1,5}$
}

1. Department of Psychiatry, Psychotherapy and Psychosomatics, Medical Faculty, Uniklinik RWTH Aachen, Aachen, Germany

2. Institute of Systems Neuroscience, Medical Faculty, Heinrich Heine University Düsseldorf, Düsseldorf, Germany

3. Institute of Neuroscience and Medicine, Brain \& Behaviour (INM-7), Research Centre Jülich, Jülich, Germany

4. RoMed Hospital Rosenheim, Department of Obstetrics, Rosenheim, Germany

5. Institute of Neuroscience and Medicine: JARA-Institute Brain Structure Function Relationship (INM 10), Research Center Jülich, Jülich, Germany

6. University Hospital, Heinrich Heine University Düsseldorf, Düsseldorf, Germany

7. Institute for Occupational, Social and Environmental Medicine, Medical Faculty, Uniklinik RWTH Aachen University, Aachen, Germany

8. Southeast Environmental Research Center, Florida International University, Florida, USA-

Corresponding author:

Susanne Stickel

Pauwelsstrasse 30

52074 Aachen

sstickel@ukaachen.de

+49241/8088734

Declarations of interest: None 
Abstract

Prolonged stress has a negative impact on the central nervous system, resulting in the vulnerability to a wide range of mental health disorders. 76 healthy postpartum mothers were studied by means of functional magnetic resonance imaging within 6 days of childbirth. The subjects were required to perform the emotional Stroop task involving happy and anxious word-face combinations. Hair samples were collected to determine cumulative hair cortisol concentration $(\mathrm{HCC})$ in the third trimester. $\mathrm{HCC}$ was found to be negatively correlated with the recruitment of the dorsal anterior cingulate cortex (ACC) and the midcingulate cortex (MCC). In response to the emotional interference of only anxious target faces, a negative correlation was seen between HCC and the bilateral orbitofrontal cortex, extending to the rostral ACC and the MCC.

Women with lower $\mathrm{HCC}$ recruited brain areas relevant to emotional cognitive control, indicating that lower HCC helps preserve conflict monitoring and resolution capacities and thus benefits mental health in pregnancy.

Keywords: postpartum period; cortisol; fMRI; emotional interference 


\section{Introduction}

Pregnancy and the postpartum period are marked by considerable changes in cortisol concentration, which is important for gestation, fetal development and parturition (I. D. Smith \& Shearman, 1974; R. Smith, Mesiano, \& McGrath, 2002). During normal pregnancy, the placenta produces and releases corticotropin-releasing hormone $(\mathrm{CRH})$ into the bloodstream, causing a dramatic rise in maternal cortisol levels (Mastorakos \& llias, 2006). In addition to the usual role of the hypothalamic-pituitary-adrenal (HPA) axis, which is embedded in a negative feedback loop to prevent further secretion of cortisol (e.g. Pariante \& Lightman, 2008; Varghese \& Brown, 2001), the additional release of CRH by the placenta leads to a positive feedback loop, enabling an increase in cortisol over the course of pregnancy (Mastorakos \& Ilias, 2006).

While normal HPA axis activity is known to be guided by the medial prefrontal cortex (mPFC), the hippocampus and the amygdala impinging on one another to inhibit stress and anxiety responses (Chattarji, Tomar, Suvrathan, Ghosh, \& Rahman, 2015), there is ample evidence to suggest that hypersecretion of cortisol due to chronic, severe or prolonged stress leads to functional neural changes with opposing effects on the hippocampus, the mPFC and the amygdala (Chattarji et al., 2015; Lucassen et al., 2014). These alterations trigger a dysregulation of the HPA axis with a cascade of subsequent deactivation patterns across a neural network including the posterior and anterior cingulate cortices (PCC, ACC), the striatum, and the insula (Pruessner et al., 2008), culminating in dysfunctional emotion regulation and impaired cognitive functions (Hamilton, Farmer, Fogelman, \& Gotlib, 2015; Price \& Drevets, 2012; Rigucci, Serafini, Pompili, Kotzalidis, \& Tatarelli, 2010).

Given that during pregnancy the placenta releases additional CRH into the blood system, a simultaneous dysregulation of the HPA axis owing to prolonged stress may render pregnant women more susceptible to neural alterations. Some longitudinal studies of the maternal brain have demonstrated that, during the postpartum period in healthy women, the brain undergoes changes leading to in- 
creased reactivity in the emotional circuits (Gingnell et al., 2015) and decreased prefrontal cortex reactivity during tasks requiring cognitive control (Bannbers et al., 2013), while in behavioral study setups, both emotion regulation and cognitive control have been seen to be affected by perinatal and postpartum changes in cortisol levels (England-Mason et al., 2017; Henry \& Sherwin, 2012). To the best of our knowledge, the investigation of the relationship between neural changes and cortisol exposure in pregnant/postpartum women has never been the subject of research, reflecting our limited knowledge of the neural mechanisms involved in the postpartum period. To test both emotional and cognitive dysregularities, tasks involving emotional distractors, such as the emotional Stroop task, have proved useful in facilitating a clear understanding of the basic mechanisms of emotional interference in cognitive control. Variations of the emotional Stroop task have been used to examine stress response and the link between the psychosocial stress elicited by negative stimuli and increased salivary cortisol levels (Hänsel \& Von Känel, 2013; Roelofs, Bakvis, Hermans, van Pelt, \& van Honk, 2007; van Honk et al., 2000).

However, with respect to pregnancy and the postpartum period, there is still uncertainty as to the precise ways in which different $\mathrm{CRH}$ activities correlate with detrimental outcomes. A recent systematic review has unveiled inconsistencies in the available literature with respect to, for instance, the correlations between high/low cortisol plasma/salivary levels and depressive outcome during pregnancy and lengths of postpartum periods, which vary across studies (Seth, Lewis, \& Galbally, 2016). The most salient factor contributing to these inconsistencies is the determination of cortisol levels by means of acute cortisol response (Seth et al., 2016). Cortisol level measurement using plasma, salivary or urinary samples is highly dependent on the circadian rhythm or individual day-to-day variability and thus mirrors only the acute cortisol levels (Kudielka, Hellhammer, \& Wüst, 2009). It has been suggested that the single cortisol measurement reflects only a situational context underlying the circadian rhythm and acute single stressors (Stalder et al., 2012). Hair cortisol concentration (HCC) measurements, on the other hand, are a promising method to examine trait estimates of cortisol during an extended period of stress and thus analyze cumulative exposure to cortisol by eliminating 
the day-to-day variability. Not surprisingly, therefore, a fair amount of recent research has focused on the effect of long-term cortisol exposure on maternal psychopathology and hair cortisol concentration (HCC) in the first and third trimesters (e.g. Braig et al., 2016; Caparros-Gonzalez et al., 2017; Scharlau et al., 2017).

In light of these observations, the overarching goal of the present work was to investigate the neural and behavioral responses of healthy postpartum women shortly after childbirth to the emotional Stroop task, and to associate the neural patterns with the cumulative cortisol exposure in the third trimester of pregnancy. Applying the emotional Stroop task to healthy postpartum women, we expected to see a link between the activation of regions responsible for emotional conflict regulation and the cumulative cortisol exposure during the last trimester of pregnancy (via HCC).

\section{Methods and materials}

\subsection{Participants}

76 healthy women were recruited at the Department of Gynecology and Obstetrics at the University Hospital Aachen within one to six days of childbirth from an ongoing longitudinal study. Women with current abuse of alcohol, drugs, psychotropic substances, antidepressant or antipsychotic medication during pregnancy, history of psychosis or manic episodes, a clinically significant depression during the pregnancy or intake of antidepressants during pregnancy were excluded from this study. Mothers of infants with genetic defects (e.g. trisomies), premature birth (less than 29 weeks of gestation) or very low birth weight (less than $1450 \mathrm{~g}$ ) were also excluded. Prior to inclusion in the study, written informed consent was obtained from each subject. The study protocol was in accordance with the Declaration of Helsinki and approved by the Institutional Review Board of the Medical Faculty, RWTH Aachen University. On the day of recruitment, anamnestic information (demographic information, information about the pregnancy, and individual and familial psychiatric history) was collected. Current depressive symptoms were assessed based on the Edinburgh Postnatal Depression Score (EPDS; 
Cox, Holden, \& Sagovsky, 1987), and a record of the number and nature of stressful life events was obtained via the Stressful Life Events Questionnaire (SLESQ; Goodman, Corcoran, Turner, Yuan, \& Green, 1998). For the fMRI measurement, an additional control for the corresponding exclusion criteria was assessed. The fMRI measurement was conducted within one to six days of childbirth depending on the day of recruitment. Prior to the measurement, women were asked to rate their current stress level on a 10-point Likert scale ( $1=$ no feelings of stress, $10=$ very stressful).

\subsection{Hair collection and laboratory measurement of hair cortisol}

In our sample, immediately after recruitment, a hair strand was cut with scissors close to the scalp from a posterior vertex position in order to assess cortisol release over the past three months (the third trimester). With the average hair growth deemed to be $1 \mathrm{~cm} /$ month (Wennig, 2000), the first 3 $\mathrm{cm}$ of the hair segment (beginning with the hairline) was used to analyze the cortisol level of the third trimester. The strand was wrapped in tinfoil to prevent additional contamination and sent to the Institute of Occupational Medicine of the University Hospital RWTH Aachen for analysis by means of the automatized online SPE LC-MS-Method (Quinete, Bertram, Reska, Lang, \& Kraus, 2015).

For the SPE LC-MS ${ }^{3}$ Method (Quinete et al., 2015), hair is minced and washed with $2.5 \mathrm{mg}$ isopropanol to remove contaminations and non-bloodborne cortisol coated on the surface of hair strands. After drying overnight at room temperature, internal standards cortisol- $d^{4}$ was added and the hair strands were incubated in $2 \mathrm{ml}$ methanol. Samples were then centrifuged at $4500 \mathrm{rmp}$ for $10 \mathrm{~min}$ and $500 \mu \mathrm{L}$ of supernatant was transferred. Chromatographic separation was carried out on an LC system (Agilent Technologies1200 Infinity series) and the cleanup step prior to analytical separation by a Poroshell C18 by ESI-MS3. The mass spectrometry was performed with a Q-Trap 5500 mass spectrometer (ABSciex, Darmstadt, Germany), with a negative ionization mode at -4500 Volt. A detailed description of the chemical processes during the analyses with SPE LC-MS ${ }^{3}$ and method validation is provided in Quinete et al. (2015). 


\subsection{Emotional Stroop-task}

The Stroop task is used to demonstrate the interference conflict and to show that trained actions are almost automatic, while unfamiliar actions require more attention. The behavioral interference (i.e. slowing down in the processing of semantically incongruous information) is observed insofar as attention resources are required to inhibit faster automatic responses (e.g. reading words) in favor of slower voluntary responses (e.g. naming colors) (Williams, Mathews, \& MacLeod, 1996). The emotional analogue of the Stroop task is often utilized to study psychiatric disorders (e.g. Chechko et al., $2012,2013,2016)$ by provoking interference through the semantic incompatibility between an emotional target (emotional face) and an emotional distractor (emotionally salient word), as the individual's current (mental) condition is reflected in a disproportionate reaction for negative (relevant to the own condition) compared to positive (irrelevant to the own condition) emotional stimuli (Williams et al., 1996). Here, the less familiar task is the recognition of the facial expression, which has to be enhanced by suppressing the habitual response like reading. In the present study, the emotional Stroop-task was presented with Presentation software (Neurobehavioral Systems, San Francisco, USA) and was displayed on an MRI-compatible BOLDscreen, visible via a mirror mounted to the head coil, during fMRI scanning. The task consisted of 120 single trials with an emotional face in the background (happy or fearful expression) and the words "ANGST" or "GLÜCK" (German for "FEAR" and "HAPPINESS") printed across the face in capital bold red letters as distractors (Figure 1). Depending on the word-emotion combinations, trials were categorized as congruent or incongruent and the number of word-emotion combination was counterbalanced across the whole run. Images of faces in normalized brightness were taken from the set used in Facial Emotions for Brain Activation (FEBA) test (Gur et al., 2002) and were put in standardized positions of the eyes and the mouth. In total, 26 different faces were used (13 happy faces [7 female], 13 fearful faces [7 female]). Each trial was presented for $1000 \mathrm{~ms}$ with a subsequent varying interstimulus interval (ISI) of 3000-5000 ms. During ISI a fixation cross was shown. To avoid priming effects, direct repetitions of the same emotion-word- 
distractor combination were avoided. The participants were asked to identify the emotion of the target face, while ignoring the word, and pressing the index (happy face) or middle finger (fearful face), using the LUMItouch response system (LumiTouch, Photon Control, Burnaby, Ca nada).

\section{0 trials}

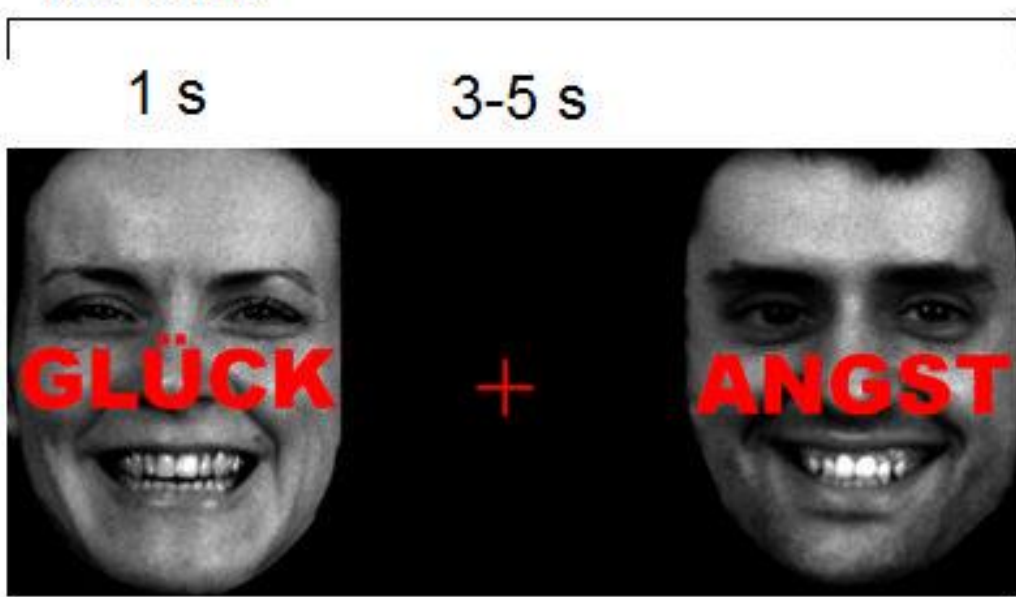

\section{Congruent}

\section{Incongruent}

Figure 1. Emotional interference task. Stimulus material consisted of congruent and incongruent facial emotion/word pairs from the FEBA faces collection.

\subsection{MRI data acquisition}

Neuroimaging data were acquired using a 3T Prisma MR scanner (Siemens Medical Systems, Erlangen, Germany) located in the Medical Faculty RWTH Aachen University. Functional images were collected with an echo-planar imaging (EPI) T2*-weighted contrast sequence sensitive to blood-oxygenlevel-dependent (BOLD) contrast (voxel size: $3.0 \times 3.0 \times 3.0$ mm³, 64 x 64 matrix, FoV: 192 x 192 mm², 34 slices, whole brain acquisition, interleaved, gap $0.75 \mathrm{~mm}, \mathrm{TR}=2020 \mathrm{~ms}, \mathrm{TE}=28 \mathrm{~ms}$, alpha $\left.=77^{\circ}\right)$. T1-weighted structural images were acquired by means of a three-dimensional magnetization-prepared rapid acquisition gradient echo image (MPRAGE) sequence (voxel size: $1 \times 1 \times 1 \mathrm{~mm}, 256 \times 256$ matrix, FoV: $256 \times 256 \mathrm{~mm}^{2}, 176$ slices, $\mathrm{TR}=2300 \mathrm{~ms}, \mathrm{TE}=1.99 \mathrm{~ms}$, alpha $\left.=9^{\circ}\right)$. 


\subsection{Analysis of behavioral data}

During the fMRI experiment, reaction times (RT) of the given answers were collected, with wrong answers and omissions being excluded from the RT analysis. For accuracy calculation, all types of errors were considered. In order to assess the effect of the facial emotion or the distractor word, two separate repeated measures analyses of variance (ANOVA) were conducted. For a 2-way emotion $x$ congruency repeated measures ANOVA, items were assigned to each level of the factors emotional face (happy or sad face) and congruency (congruent or incongruent). For a 2-way distractor word $\mathrm{x}$ congruency, items were assigned to each level of the distractor word ("ANGST" or "GLUECK") and congruency (congruent or incongruent). An additional 2-way target/distractor $x$ emotion repeated measures ANOVA with the levels target or distractor and emotion (happy or anxious) was conducted in order to assess the task-distractor interaction.

The interference effect is defined as the difference of RT (or accuracy) in incongruent trials vs. congruent trials.

\subsection{FMRI data analysis}

Image preprocessing: Images were processed using the Statistical Parametric Mapping (SPM) software (version SPM8, http:// www.fil.ion.ucl.ac.uk/spm) based on Matlab 2015b (Mathworks, Inc., Natick, Massachusetts, United States). The first four images of each time series were excluded due to T1 stabilization effects. All remaining images were slice-time corrected and realigned to the first functional image. Images were normalized into Montreal Neurologic Institute (MNI) space (resampling to $2 \times 2 \times 2 \mathrm{~mm} 3$ ) and smoothed with an isotropic Gaussian kernel (8mm full width at half maximum).

For each subject, delta functions with the time-points of trial presentation of each type were convolved with the canonical hemodynamic response function (HRF) to build regressors for the time series model. For each task, a first-level model was estimated including four regressors of interest: 2 
levels of conflict (congruent vs. incongruent) by the 2 levels of each emotional face (happy face vs. anxious face). The first-level model also included an additional (HRF-convolved) regressor of no interest for error trials (wrong answers and omissions) and an intercept for the mean across each session. A high-pass filter with a cut-off period of $128 \mathrm{~s}$ was applied and serial auto-correlations were accounted for by including a first-order auto-regressive covariance structure (AR(1)). Three contrasts were computed at individual levels: (1) all incongruent trials > all congruent trials, (4) only anxious faces: incongruent trials > congruent trials, (5) only happy faces: incongruent trials $>$ congruent trials. An SPM8 random-effects analysis was performed by entering the contrasts into separate one-sample t-tests and a whole-brain multiple regression analysis. The statistical threshold of the one-sample ttests, unless not otherwise mentioned, was set at $p<.05$ cluster-level FWE-correction, with a clusterforming threshold at voxel-level $p<.001$. The same was true for the correlation analyses, which were performed in order to determine the relationship between activation strength in response to incongruent (as compared to congruent) trials in single-subject contrasts on the one hand and individual hair cortisol levels on the other. Follow-up correlational analysis were performed by extracting mean activation parameters from significant whole-brain results.

\section{Results}

\subsection{Descriptive statistics}

Descriptive information of the study sample $(n=76)$ is presented in Table 1 . The average postpartum time frame of measurement was 3 days after childbirth $(M=2.92$ days, $S D=1.14$ days). The current stress feelings before the measurement followed an even distribution without any particular accumulation of a certain stress value (range $1-10, M=4.80, S D=2.69$ ), and no correlation was observed between HCC and currents stress feelings $(r=.32, p=.786)$ and EPDS $(r=-.056, p=.628)$. Additional correlations of the descriptive data can be found in the Supplements. 
Additionally, correlations between HCC and any of the other obtained variables were not significant after Bonferroni correction for multiple testing.

Table 1

Sample characteristic, means (M) and standard deviations (SD).

\begin{tabular}{lll}
\hline & M (SD) & Percent \\
\hline Age & $30.78(4.69)$ & \\
Depressive symptoms (EPDS) & $4.99(3.78)$ & \\
Number of personal stressful life events & $1.11(1.55)$ & 31.6 \\
Gestational age in days & $271.99(15.55)$ & 25 \\
Weight of child in grams & $3247.37(654.3)$ & 32.9 \\
Hair cortisol concentration in mg & $10.84(15.59)$ & \\
Infant relocation to infant ward & & \\
Psychiatric history prior to pregnancy & & \\
Familial psychiatric history & & \\
\hline
\end{tabular}

Note. EPDS = Edinburgh Postnatal Depression Scale.

\subsection{Behavioral results}

There was a significant effect of the factor congruency $\left(F(1,87)=162.35 . p<.001\right.$. eta $\left.{ }^{2}=.651\right)$, reflecting faster RT in congruent compared to incongruent trials (Figure 2A). There was also a significant effect of the factor emotional face $\left(F(1,87)=26.11, p<.001\right.$. eta $\left.^{2}=.231\right)$, showing that anxious faces $(M=785.03 \mathrm{~ms}, \mathrm{SE}=18.76)$ were processed more slowly than happy faces $(\mathrm{M}=742.37 \mathrm{~ms}, \mathrm{SE}=$ 19.38). Similarly, RTs were slower $\left(F(1,87)=13.08, p<.001\right.$, eta $\left.^{2}=.131\right)$ when the distractor word was anxious ("ANGST", M = $769.66 \mathrm{~ms}, \mathrm{SE}=18.38$ ) compared to the distractor word happy ("GLÜCK", $M=757.73 \mathrm{~ms}, \mathrm{SE}=18.99)$. 
There was also an emotion $x$ word interaction $\left(F(1,87)=13.95 . p<.001\right.$. eta $\left.^{2}=.138\right)$ (Figure 2B). In the happy condition, words were processed more slowly than faces, while in the anxious condition, faces were processed more slowly than words. The RT differences between incongruent and congruent trials were compared across all emotions. A dependent t-test revealed that interference was increased for happy $(M=62.86, S D=53.77)$ compared to anxious faces $(M=39.01, S D=42.82, t(87)=-$ $3.62, \mathrm{p}<.001)$.

For accuracy analysis, the 2-way face $\mathrm{x}$ congruency repeated measures ANOVA revealed a significant effect of congruency $\left(F(1,87)=72.79, p<.001\right.$, eta $\left.^{2}=.456\right)$ and of emotion $(F(1,87)=5.40, p=.022$, eta $\left.^{2}=.058\right)$. Bonferroni-corrected pairwise comparison indicated a higher level of correct responses during congruent compared $(96.9 \%)$ to incongruent $(93.9 \%)$ trials $(p<.001)$ and greater accuracy for happy $(96.5 \%)$ compared to anxious $(96.5 \%)$ faces $(p=.022)$.
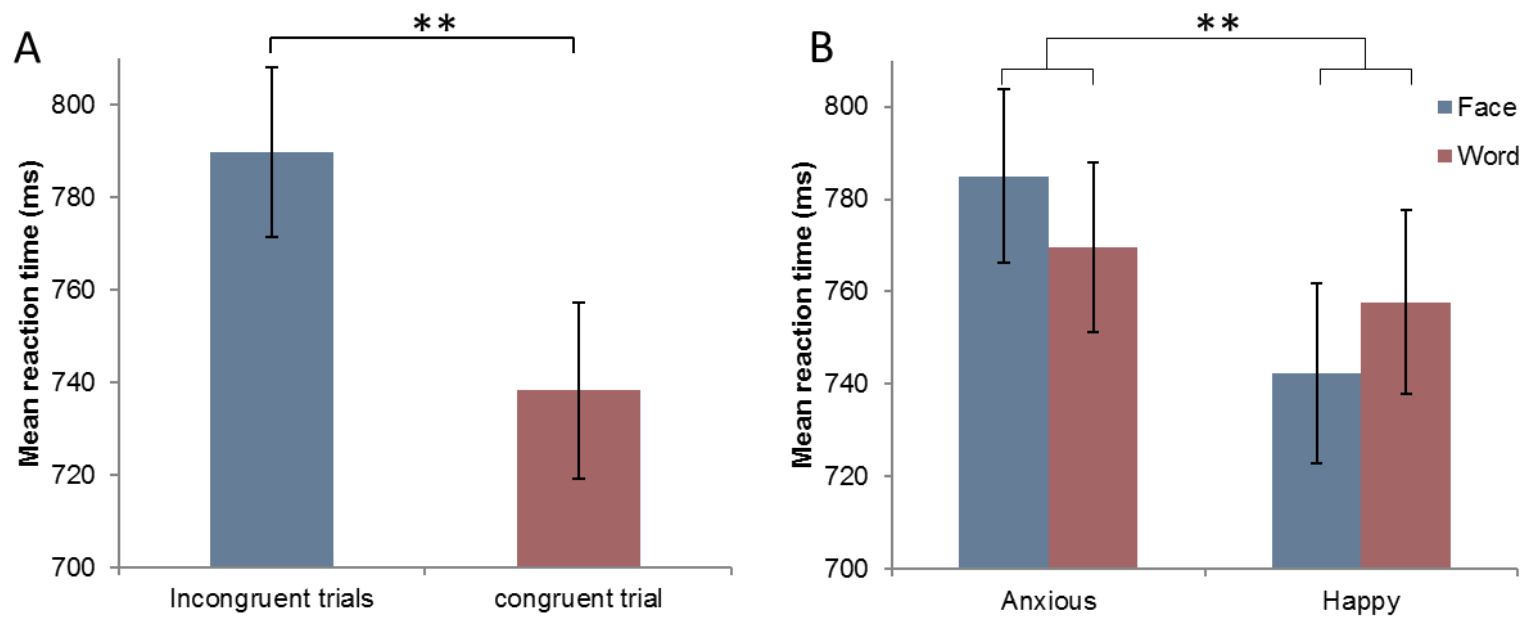

Figure 2. Mean reaction time (RT) and standard deviation (error bars) A) Conflict effects across all four conditions. RTs to incongruent and anxious trials were significantly slower than RTs to congruent and happy trials, respectively. B) Interaction of target face and distractor word. RTs to happy words were significantly slower than to happy faces. In the anxious condition, words were processed significantly faster than faces. ${ }^{* *} p<.001$

There were no other significant results and no significant correlations between the RTs and HCC. 


\section{3 fMRI results}

\subsubsection{Effect of emotional conflict on BOLD response}

At a whole brain at $p<.05$ cluster-level FWE-correction, with a cluster-forming threshold at voxellevel $p<.001$, in response to incongruent compared to congruent trials, we found a significant BOLD signal increase in a cluster (50.541 voxel) encompassing the bilateral IFG (left: p. orbitalis; peak MNI: $-38 / 22 /-6, T=7.72$; p. opercularis; peak MNI: $-50 / 10 / 28, T=6.98$; right: p. triangularis; peak MNI: $46 / 20 / 2, T=7.52$ ) and the bilateral insula (right: peak MNI -34/18/-8, T = 7.9; right: peak MNI: $34 / 28 /-4, T=7.97)$, extending to the bilateral dorsal $A C C$, the midcingulate cortex $(M C C)$, the precentral gyrus, and the superior medial and middle frontal gyri. Another large cluster (1821 voxel) comprised the right supramarginal gyrus (peak $\mathrm{MNI}: 50 /-38 / 44, \mathrm{~T}=4.35$ ), the right middle occipital gyrus (peak MNI: 34/-68/26, T = 4.20) and the right angular gyrus (peak MNI: 30/-58/38, T = 4.07), extending to the right fusiform gyrus, the bilateral cerebellum, the left superior and inferior parietal gyri, the bilateral calcarine gyrus as well as the left lingual gyrus. The results are presented in Figure 3.
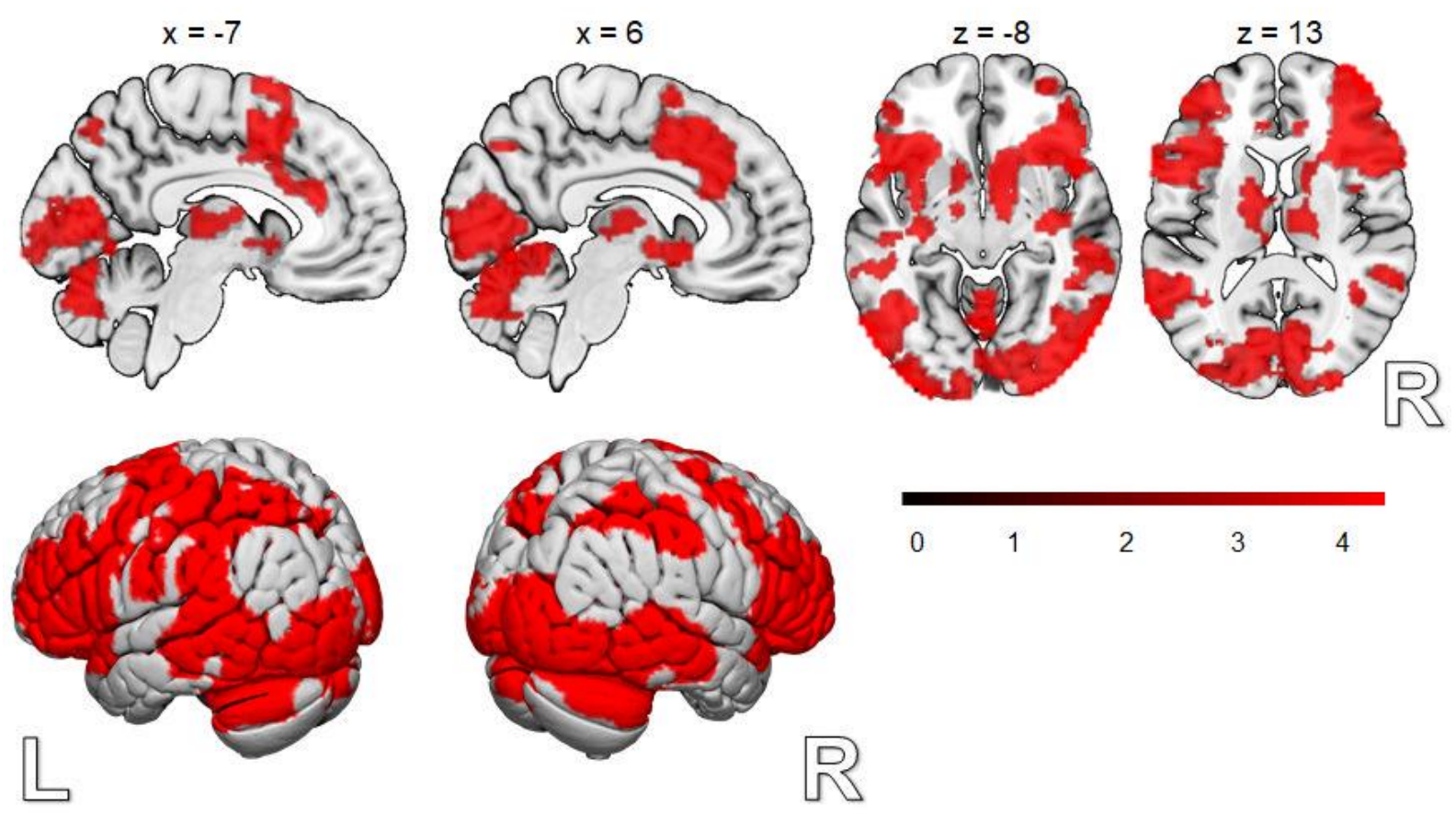
Figure 3. Brain regions activated during the emotional conflict (incongruent $>$ congruent trials). Activity is shown at a $p<.05$ cluster-level FWE-correction, with a cluster-forming threshold at voxel-level $p$ $<.001$.

With an analysis at $\mathrm{p}<.05$ cluster-level FWE-correction, using a cluster-forming threshold at voxellevel $p<.001$, comparing the interference effect of only happy faces, we found a significant BOLD signal increase in the bilateral insula, extending to the IFG, the MCC extending to the ACC, and the bilateral temporal and right parietal cortices (see Table 2).

Analyzing the interference effect of only anxious target faces, we found a significant BOLD signal increase in the bilateral occipital cortex and the left parietal cortex. Additionally, significant clusters were seen to have emerged involving the bilateral IFG/insula, the right MCC and the posterior medial frontal gyrus (see Table 2).

Table 2

Brain regions recruited more strongly in response to the emotional conflict of only anxious and only happy faces (incongruent $>$ congruent trials), $p<.05$ cluster-level FWE-correction, with a clusterforming threshold at voxel-level $p<.001$.

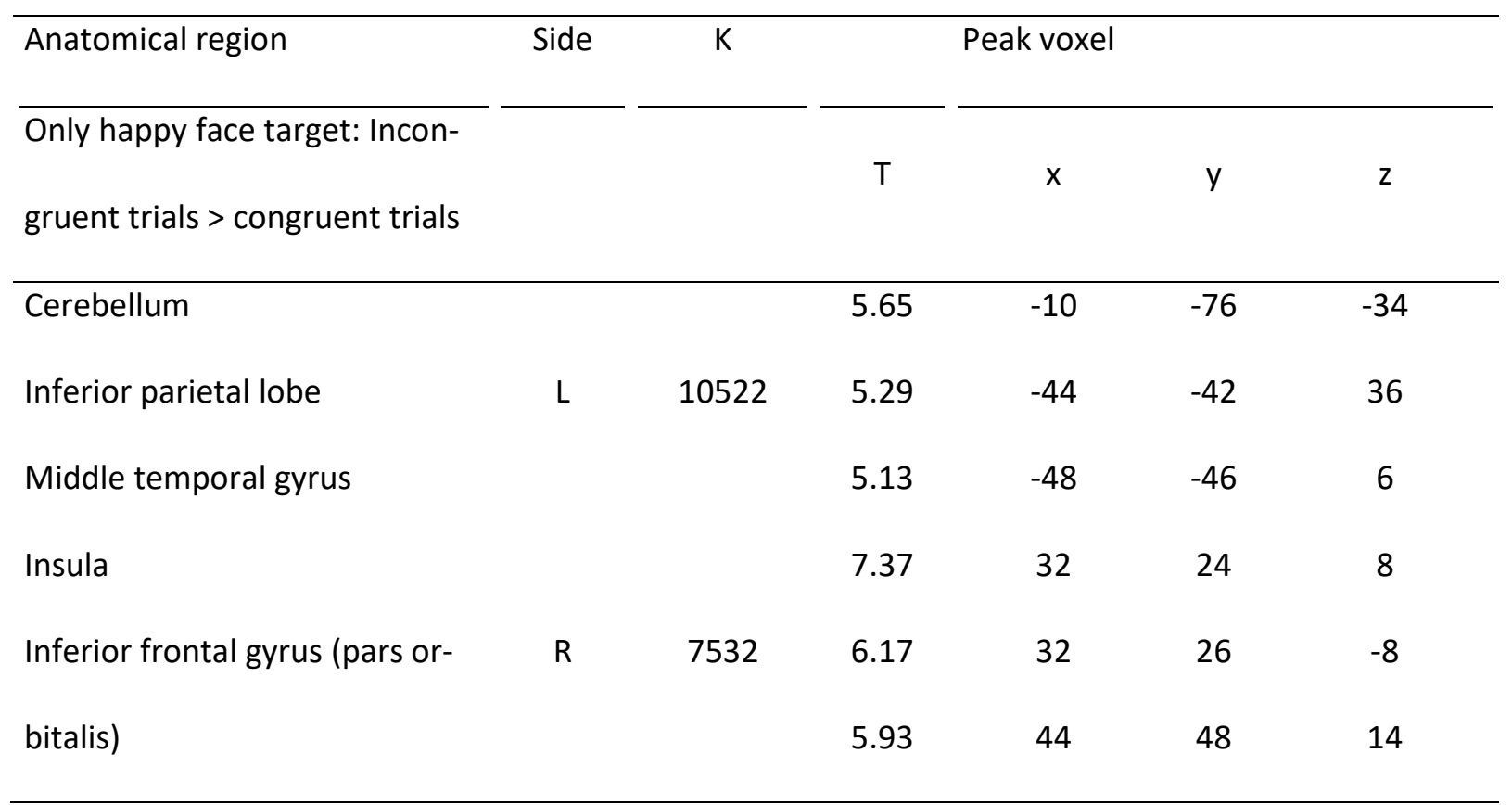


Middle frontal gyrus

Insula

Inferior frontal gyrus (pars

$\begin{array}{llll}8.17 & -38 & 20 & -4\end{array}$

opercularis)

$L$

6484

6.40

$-48$

6

28

Inferior frontal gyrus (pars tri-

5.73

$-40$

12

24

angularis)

Midcingulate cortex

$\mathrm{R}$

5.87

10

24

32

Anterior cingulate cortex

2439

Superior parietal lobe

R

5.41

14

26

26

Inferior parietal lobe

1315

Middle temporal gyrus

$R$

$\begin{array}{llll}5.05 & 52 & -40 & 58\end{array}$

Inferior temporal gyrus

735

4.48

48

$-48$

42

Thalamus

4.69

54

$-34$

0

3.48

54

$-34$

18

5.38

$-14$

$-12$

8

Caudate nucleus

L

542

4.16

$-10$

12

$-4$

Pallidum

3.92

$-10$

6

$-4$

Inferior occipital gyrus

4.68

$-46$

$-68$

$-12$

Inferior temporal gyrus

4.40

$-42$

$-62$

$-10$

Only anxious face target: Incon-

gruent trials $>$ congruent trials

\begin{tabular}{|c|c|c|c|c|c|c|}
\hline Inferior occipital gyrus & & & 6.73 & 40 & -90 & -6 \\
\hline Middle occipital gyrus & $\mathrm{R}$ & 3835 & 6.02 & 34 & -96 & 4 \\
\hline Cerebellum & & & 5.79 & 40 & -48 & -32 \\
\hline Cerebellum & & & 5.37 & -30 & -60 & -18 \\
\hline & $\mathrm{L}$ & 2559 & & & & \\
\hline Middle occipital gyrus & & & 5.11 & -36 & -92 & 4 \\
\hline
\end{tabular}




\begin{tabular}{|c|c|c|c|c|c|c|}
\hline \multicolumn{7}{|l|}{ Inferior frontal gyrus (pars tri- } \\
\hline angularis) & & & 4.58 & -34 & 18 & 10 \\
\hline Insula & L & 784 & 4.31 & -30 & 28 & 0 \\
\hline Inferior frontal gyrus (pars or- & & & 4.23 & -54 & 28 & -4 \\
\hline \multicolumn{7}{|l|}{ bitalis) } \\
\hline \multicolumn{7}{|l|}{ Insula } \\
\hline & & & 5.52 & 36 & & -4 \\
\hline Inferior frontal gyrus (pars or- & & & & & 28 & \\
\hline bitalis) & $\mathrm{R}$ & 729 & 4.77 & 54 & 2418 & -6 \\
\hline & & & 3.66 & 52 & & -14 \\
\hline \multicolumn{7}{|l|}{ Temporal lobe } \\
\hline Inferior parietal lobe & & & 4.64 & -26 & -54 & 44 \\
\hline Superior parietal lobe & $\mathrm{L}$ & 445 & 4.50 & -26 & -60 & 44 \\
\hline Angular gyrus & & & 3.74 & -28 & -52 & 34 \\
\hline Posterior medial frontal gyrus & $\mathrm{L}$ & 401 & 4.98 & -8 & 10 & 46 \\
\hline Midcingulate cortex & $\mathrm{R}$ & & 4.19 & 12 & 10 & 44 \\
\hline Precentral gyrus & L & 244 & 5.08 & -38 & -4 & 62 \\
\hline \multicolumn{7}{|l|}{ Precentral gyrus } \\
\hline & & & 4.37 & -40 & 0 & 38 \\
\hline Inferior frontal gyrus (pars & $\mathrm{L}$ & 244 & 3.67 & -40 & 4 & 22 \\
\hline opercularis) & & & & & & \\
\hline Postcentral gyrus & & & 4.53 & -34 & -36 & 44 \\
\hline & L & 200 & & & & \\
\hline Inferior parietal lobe & & & 4.27 & -44 & -34 & 38 \\
\hline
\end{tabular}

Note. $\mathrm{L}=$ left hemisphere; $\mathrm{R}=$ right hemisphere. 
3.2.2 Correlation between BOLD response to interference conflict and cumulative level of cortisol in the third trimester

At a whole-brain level at $\mathrm{p}<.05$ cluster-level FWE-correction, with a cluster-forming threshold at voxel-level $p<.001$, the modulation of incongruent trials contrasted to congruent trials showed that the right supramarginal gyrus $(r=-.55, p<.001)$, the bilateral dorsal ACC extending to right the MCC $(r=-.47, p<.001)$, the left MCC $(r=-0.48, p<.001)$, the temporal gyrus $(r=-0.54, p<.001)$, and the precuneus $(r=-.48, p<.001)$ were correlated with lower levels of HCC in the third trimester (see Table 3, Fig. 4A). There was no significant positive correlation between the interference conflict and HCC.

During the emotional interference of anxious faces, by disentangling the emotional target faces, we found a correlation between lower levels of cortisol and higher recruitment of bilateral orbitofrontal cortex (OFC), extending to the rostral ACC, the subgenual ACC and the superior frontal gyrus $(r=-.55$, $p<.001)$, the right precuneus, extending to the left PCC/MCC $(r=-.52, p<.001)$, the right MCC $(r=-$ $.44, p<.001)$, the post-/precentral gyrus $(r=-0.47, p<.001)$, the right angular gyrus, extending to the supramarginal gyrus and the middle temporal gyrus $(r=-0.52, p<.001)$, as well as the left inferior parietal lobule extending to the middle temporal gyrus and the angular gyrus $(r=-0.47, p<.001)$ (see Table 2, Figure 4B). No significant correlation was seen between the BOLD response and higher HCC.

Additionally, we did not find any significant clusters in the correlation analyses involving the emotional interference of happy faces and both higher and lower cortisol levels. The whole-brain regression analysis with the covariate subjective stress feelings prior to measurement yielded no significant brain activation either in the emotional interference of incongruent > congruent trials or in only happy and only anxious faces. Also, a whole-brain regression analysis using both subjective stress values and HCC as covariates revealed no significant thresholds in any contrast. 
Finally, we performed a follow-up analysis with the extracted mean activation of the significant regions, based on the whole brain correlation of $\mathrm{HCC}$ and the interference conflict and the whole brain correlation of HCC and the interference of anxious faces. We did not observe any significant correlations with RT or current stress feelings in either contrast.

Table 3

Whole-brain condition effects negatively correlated with cumulative hair cortisol, with $p<.05$ cluster-level FWE-correction, with a cluster-forming threshold at voxel-level $p<.001$. For each cluster, a maximum of three significant regions in gray matter is reported.

\begin{tabular}{|c|c|c|c|c|c|c|}
\hline \multirow[t]{2}{*}{ Anatomical region } & \multirow[t]{2}{*}{ Side } & \multirow[t]{2}{*}{ k } & \multicolumn{4}{|c|}{ Peak voxel } \\
\hline & & & $\mathrm{T}$ & $x$ & Y & Z \\
\hline \multicolumn{7}{|c|}{$\begin{array}{l}\text { Happy and anxious face targets: In- } \\
\text { congruent trials > congruent trials }\end{array}$} \\
\hline Supramarginal gyrus & $\mathrm{R}$ & 678 & 5.42 & 64 & -58 & 36 \\
\hline Anterior cingulate gyrus & L & & 4.39 & -6 & 32 & 16 \\
\hline Midcingulate gyrus & $\mathrm{R}$ & 381 & 4.06 & 8 & 20 & 30 \\
\hline Anterior cingulate gyrus & $\mathrm{R}$ & & 3.83 & 8 & 30 & 24 \\
\hline Midcingulate gyrus & L & 291 & 4.63 & -4 & -32 & 48 \\
\hline Middle temporal gyrus & L & 278 & 4.88 & -56 & -48 & 6 \\
\hline Angular gyrus & L & 277 & 4.20 & -56 & -54 & 32 \\
\hline \multirow[t]{2}{*}{ Inferior parietal lobule } & L & & 4.00 & -48 & -50 & 36 \\
\hline & L & & 4.68 & -12 & -54 & 44 \\
\hline \multirow[t]{2}{*}{ Precuneus } & & 259 & & & & \\
\hline & $\mathrm{R}$ & & 4.33 & 16 & -54 & 44 \\
\hline
\end{tabular}

Only anxious face target: Incongruent trials $>$ congruent trials 
Cumulative cortisol secretion in pregnancy

\begin{tabular}{|c|c|c|c|c|c|c|}
\hline Superior frontal gyrus & $\mathrm{R}$ & & 6.02 & 16 & 26 & 56 \\
\hline Middle orbital gyrus & $\mathrm{L}$ & 3021 & 5.74 & 0 & 44 & -6 \\
\hline Rectal gyrus & L & & 5.74 & -2 & 44 & -10 \\
\hline Precuneus & $\mathrm{R}$ & & 5.21 & 4 & -54 & 24 \\
\hline Posterior cingulate cortex & L & 1514 & 5.06 & 6 & -48 & 22 \\
\hline Midcingulate cortex & L & & 4.83 & 2 & -48 & 36 \\
\hline Angular gyrus & $\mathrm{R}$ & & 6.08 & 60 & -58 & 36 \\
\hline Supramarginal gyrus & $\mathrm{R}$ & 683 & 4.71 & 54 & -52 & 34 \\
\hline Middle temporal gyrus & $\mathrm{R}$ & & 5.13 & 58 & -66 & 26 \\
\hline Inferior parietal lobule & L & & 4.58 & 30 & -82 & 48 \\
\hline Middle temporal gyrus & L & 549 & 4.13 & -48 & -66 & 22 \\
\hline Angular gyrus & L & & 3.91 & -42 & -76 & 36 \\
\hline Midcingulate cortex & $\mathrm{R}$ & 276 & 4.53 & 8 & -22 & 40 \\
\hline \multirow[t]{2}{*}{ Postcentral gyrus } & $\mathrm{L}$ & & & -50 & -10 & 44 \\
\hline & & 248 & 4.76 & & & \\
\hline Precentral gyrus & L & & & -52 & -12 & 36 \\
\hline
\end{tabular}

Note. $L=$ left hemisphere; $R=$ right hemisphere. 

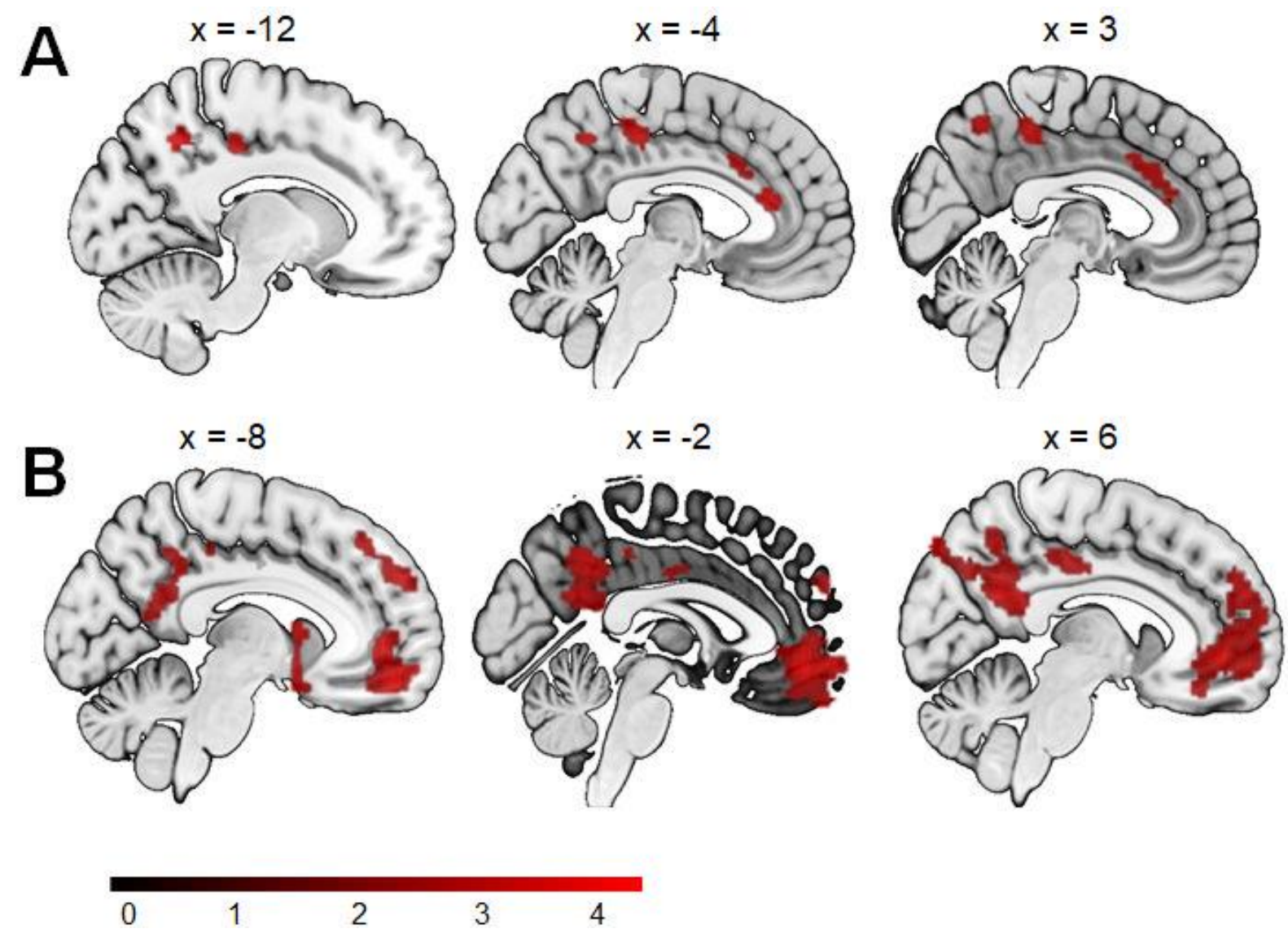

Figure 4. Whole-brain correlation between neural activation and cumulative cortisol levels (HCC) in the third trimester A) Whole-brain correlation between HCC and areas recruited during the emotional interference task (incongruent > congruent trials). B) Whole-brain correlation between HCC and areas recruited during emotional interference of anxious faces (incongruent $>$ congruent trials). Activity is shown at a $\mathrm{p}<.05$ cluster-level FWE-correction, with a cluster-forming threshold at voxellevel $p<.001$

\section{Discussion}

In the emotional Stroop task applied in the study, using face stimuli as targets and word stimuli as distractors, the subjects, healthy postpartum women within a few days of childbirth, reacted more slowly and less accurately to incongruent compared to congruent trials, with the interference conflict (incongruent > congruent trials) being greater for the happy target faces than the anxious ones. Addi- 
tionally, the effect of emotion became evident as anxious faces as well as the word "ANGST" (anxiety), compared to happy faces or the word "GLÜCK" (happiness), led to both slower RT and lower accuracy. There were no significant correlations between HCC and RT and lower accuracy or any other parameter obtained by the participants.

Slower RT for anxious faces can be construed as a positive attention bias in favor of approaching a cue rather than avoiding it (Mathews \& MacLeod, 1984). Vigilance to negative stimuli is associated with increased levels of salivary cortisol following a selective attention task (van Honk et al., 2000), with parallels to this phenomenon seen in artificial stress induction and the subsequent increase in salivary cortisol (Roelofs et al., 2007; Roelofs, Elzinga, \& Rotteveel, 2005), and the link between childhood maltreatment and increased salivary cortisol in response to emotional Stroop task in postpartum women 7 months after childbirth (England-Mason et al., 2017). While only acute stress responses are reflected in salivary cortisol levels, HCC measurement helps assess prolonged stress exposure over a retrospective timeframe of three months. Prior to the fMRI measurement, our participants had been asked to rate their current perceived stress, although no correlation was found between the self-reported stress and either HCC or the Stroop task RT. Various studies have described a poor covariance of self-reported stress and HPA axis activity measured by salivary stress (for a review, see Hellhammer, Wüst, \& Kudielka, 2009; Kudielka et al., 2009), which is likely due to different timing and dynamics of the systems (Schlotz et al., 2008). Based on this, we suggest that the absence of significant correlations between perceived stress and the behavioral data from the Stroop task is due to the lack of psychoendocrine covariance (Schlotz et al., 2008). We also maintain that the non-significant correlations between HCC and the behavioral data are on account of the retrospective measurement of a three-month stress exposure by HCC, which does not represent an acute endocrine stress response associated with the negative attention bias found in other studies (EnglandMason et al., 2017; Roelofs et al., 2007, 2005; van Honk et al., 2000).

However, the prolonged stress measured by HCC and the additional CRH secreted by the placenta in the third trimester have an influence on the activity of the HPA axis with respect to the relevant brain 
regions. In the present study, the involvement of regions known to be linked to cognitive emotion regulation, e.g. the supramarginal gyrus, the dorsal ACC/MCC, the middle temporal gyrus, the angular gyrus/inferior parietal cortex and the precuneus (Kohn et al., 2014), were negatively correlated with HCC levels in the third trimester. The analysis of interference only during anxious trials showed $\mathrm{HCC}$ to be negatively correlating with a network including the mPFC extending to the rostral ACC and parts of the subgenual ACC as well as the MCC region, the PCC and the precuneus. According to a significant body of research, the MPFC is involved in approach-avoidance behavior (for a review, see Spielberg, Stewart, Levin, Miller, \& Heller, 2008), with lateralized activation of the right PFC for avoidance and the left PFC for approach (Sutton \& Davidson, 1997). Additionally, the mPFC is one of the key target areas for the HPA axis-elicited changes in cortisol release, allowing prolonged stress exposure to alter the functional properties of the region (Chattarji et al., 2015). To the best of our knowledge, no previous study has investigated the link between the three-month-long stress exposure measured via HCC and the neural activation of the MPFC. Considering that HCC is a reliable measure and a retrospective biomarker of HPA activity (D'Anna-Hernandez, Ross, Natvig, \& Laudenslager, 2011; Meyer \& Novak, 2012), and that it is ineffective in detecting short-term stressors such as salivary cortisol, we would like to conclude that the long-term effect of cortisol on brain regions measured by HCC is better suited to retrospectively predict neuronal task-based activation than to explain behavioral results.

Both attentional and cognitive control of emotions are suggested to be regulated by the mPFC (Cieslik, Mueller, Eickhoff, Langner, \& Eickhoff, 2015; Kohn et al., 2014). Human and animal lesion studies have emphasized the role of the MPFC in initiating and maintaining task-relevant response (Stuss \& Alexander, 2007) as well as emotion-influenced behavior (Roberts \& Wallis, 2000). Involved in conflict monitoring, behavioral adjustment and executive control (e.g. Kerns et al., 2004), the dorsal ACC is often rather cognitively motivated (e.g. Chechko et al., 2012; Kerns et al., 2004), while the rostral ACC is particularly involved in the resolution of emotional conflict, i.e. controlling emotionally incompatible stimuli and one's own emotional response (Etkin, Egner, Peraza, Kandel, \& Hirsch, 
2006). In addition, regions of the rostral ACC, including the subgenual ACC and the OFC, have been shown to be involved in depression (Drevets et al., 1997; Price \& Drevets, 2012), while the dorsal ACC is impaired in stress-related disorders (e.g. Chechko et al., 2009). For instance, in postpartum mothers (Laurent \& Ablow, 2012, 2013), hypoactivation of the dorsal ACC, seen in response to cry sounds and the distressed face of their own infant, has been linked to an increase in depressive symptoms. Also, as previous research has shown, higher salivary cortisol levels increase selective attention towards negative stimuli (Hänsel \& Von Känel, 2013; Roelofs et al., 2007; van Honk et al., 2000), with one imaging study finding healthy subjects dosed with hydrocortisone displaying a deactivation of the subgenual ACC in response to sad stimuli (Sudheimer et al., 2013). In our sample of post-childbirth healthy mothers, the negative correlation observed between the $\mathrm{HCC}$ values and the recruitment of the MPFC including the dorsal ACC and the rostral ACC may therefore indicate that, in those who had more stress in the last trimester of pregnancy, neural changes might have occurred in the regions relevant to conflict detection, conflict resolution, cognitive control over emotional stimuli as well as self-reflection and semantic processes linked to identifying the emotional value of stimuli. This in turn may suggest that these women are more susceptible to impaired stress resistance and, therefore, are likely to be less capable of resolving anxious emotions shortly after giving birth.

Finally, the anterior part of the MCC is known to be involved in both attentional and cognitive emotion control functions (Cieslik et al., 2015; Kohn et al., 2014), playing a central role in connecting cognitive control and affect, especially when confronted with negative emotions or ambiguous stimuli which provoke uncertainty in action (Shackman et al., 2011), as well as during interference tasks with emotional words (K. D. Davis et al., 2005). To the best of our knowledge, no systematic research exists addressing the issues of either cortisol influence on MCC activity or the role of the MCC in perinatal/postpartum depression. We suggest that lower levels of cortisol in the third trimester facilitate the detection of ambiguous stimuli when interfered with negative emotions. As the Stroop task reflects a mother's own mental condition through the reaction to negative compared to positive stimuli 
(Williams, Mathews, \& MacLeod, 1996), lower HCC in pregnancy may be beneficial for mental health in the postpartum period.

The relationship between inhibitory behavior and emotion processing is of great importance in the context of the vulnerability to mood disorders. A number of studies have shown that difficulties in cognitive control ensuing from failed inhibition of negative stimuli can enhance ruminative behavior in both healthy individuals and depressed patients (R. N. Davis \& Nolen-Hoeksema, 2000; Joormann \& Gotlib, 2010). It is possible that women with higher HCC, who showed the inverse correlation between HCC and activation patterns in response to the emotional Stroop task in our study, may be more susceptible to developing depression. While our subjects were identified as healthy at the time of recruitment (within 1-6 days of childbirth), in the general population of postpartum mothers, approximately $10-20 \%$ (O'Hara \& McCabe, 2013) have been found to develop a postpartum depression (PPD) within two to six weeks of childbirth, with an estimated $50 \%$ of the cases remaining unidentified (Chaudron et al., 2001). Early diagnosis and tailored interventions can help reduce the risk and limit the damage of PPD (Dennis \& Dowswell, 2013), hence the need for more research in the area of PPD.

One limitation of the study, however, is that it fails to ascertain if the observed HCC-dependent activation patterns within the $\mathrm{mPFC} /$ dorsal $\mathrm{ACC}$ have any clinical relevance in terms of predisposing mothers to postpartum depression. This shortcoming notwithstanding, to our knowledge, this is the first study to examine the association between HCC levels in the third trimester of pregnancy and neural response to emotional interference shortly after childbirth. Accurate identification of PPD risk factors, however, remains a major challenge, underscoring the need for more systematic research in the field.

\section{Acknowledgments}

We thank all participants. 
Funding

This study was supported by the rotation program of the medical faculty of the University Hospital RWTH Aachen and the International Research Training Group (IRTG 2150) of the German Research Foundation (DFG). 
Reference

Bannbers, E., Gingnell, M., Engman, J., Morell, A., Sylvén, S., Skalkidou, A., ... Poromaa, I. S. (2013). Prefrontal activity during response inhibition decreases over time in the postpartum period. Behavioural Brain Research, 241, 132-138. https://doi.org/10.1016/J.BBR.2012.12.003

Braig, S., Grabher, F., Ntomchukwu, C., Reister, F., Stalder, T., Kirschbaum, C., ... Genuneit, J. (2016). The association of hair cortisol with self-reported chronic psychosocial stress and symptoms of anxiety and depression in women shortly after delivery. Paediatric and Perinatal Epidemiology, 30(2), 97-104. https://doi.org/10.1111/ppe.12255

Caparros-Gonzalez, R. A., Romero-Gonzalez, B., Strivens-Vilchez, H., Gonzalez-Perez, R., MartinezAugustin, O., \& Peralta-Ramirez, M. I. (2017). Hair cortisol levels, psychological stress and psychopathological symptoms as predictors of postpartum depression. PLOS ONE, 12(8), e0182817. https://doi.org/10.1371/journal.pone.0182817

Chattarji, S., Tomar, A., Suvrathan, A., Ghosh, S., \& Rahman, M. M. (2015). Neighborhood matters: divergent patterns of stress-induced plasticity across the brain. Nature Neuroscience, 18(10), 1364-1375. https://doi.org/10.1038/nn.4115

Chaudron, L. H., Klein, M. H., Remington, P., Palta, M., Allen, C., \& Essex, M. J. (2001). Predictors, prodromes and incidence of postpartum depression. Journal of Psychosomatic Obstetrics \& Gynecology, 22(2), 103-112. https://doi.org/10.3109/01674820109049960

Chechko, N., Augustin, M., Zvyagintsev, M., Schneider, F., Habel, U., \& Kellermann, T. (2013). Brain circuitries involved in emotional interference task in major depression disorder. Journal of Affective Disorders, 149(1-3), 136-145. https://doi.org/10.1016/j.jad.2013.01.013

Chechko, N., Kellermann, T., Augustin, M., Zvyagintsev, M., Schneider, F., \& Habel, U. (2016). Disorder-specific characteristics of borderline personality disorder with co-occurring depression and its comparison with major depression: An $\mathrm{fMRI}$ study with emotional interference task. Neurolmage: Clinical, 12, 517-525. https://doi.org/10.1016/j.nicl.2016.08.015

Chechko, N., Kellermann, T., Zvyagintsev, M., Augustin, M., Schneider, F., \& Habel, U. (2012). Brain circuitries involved in semantic interference by demands of emotional and non-emotional distractors. PLOS ONE, 7(5), e38155. https://doi.org/10.1371/journal.pone.0038155

Chechko, N., Wehrle, R., Erhardt, A., Holsboer, F., Czisch, M., \& Sämann, P. G. (2009). Unstable prefrontal response to emotional conflict and activation of lower limbic structures and brainstem in remitted panic disorder. PLOS ONE, 4(5), e5537. https://doi.org/10.1371/journal.pone.0005537

Cieslik, E. C., Mueller, V. I., Eickhoff, C. R., Langner, R., \& Eickhoff, S. B. (2015). Three key regions for supervisory attentional control: Evidence from neuroimaging meta-analyses. Neuroscience and Biobehavioral Reviews, 48, 22-34. https://doi.org/10.1016/j.neubiorev.2014.11.003

Cox, J. L., Holden, J. M., \& Sagovsky, R. (1987). Detection of postnatal depression. Development of the 10-item Edinburgh Postnatal Depression Scale. The British Journal of Psychiatry, 150, 782786. Retrieved from http://www.ncbi.nlm.nih.gov/pubmed/3651732

D’Anna-Hernandez, K. L., Ross, R. G., Natvig, C. L., \& Laudenslager, M. L. (2011). Hair cortisol levels as a retrospective marker of hypothalamic-pituitary axis activity throughout pregnancy: Comparison to salivary cortisol. Physiology \& Behavior, 104(2), 348-353. https://doi.org/10.1016/J.PHYSBEH.2011.02.041 
Davis, K. D., Taylor, K. S., Hutchison, W. D., Dostrovsky, J. O., McAndrews, M. P., Richter, E. O., \& Lozano, A. M. (2005). Human anterior cingulate cortex neurons encode cognitive and emotional demands. Journal of Neuroscience, 25(37), 8402-8406.

https://doi.org/10.1523/JNEUROSCI.2315-05.2005

Davis, R. N., \& Nolen-Hoeksema, S. (2000). Cognitive inflexibility among ruminators and nonruminators. Cognitive Therapy and Research, 24(6), 699-711. https://doi.org/10.1023/A:1005591412406

Dennis, C.-L., \& Dowswell, T. (2013). Psychosocial and psychological interventions for preventing postpartum depression. Cochrane Database of Systematic Reviews, (2), 1-102. https://doi.org/10.1002/14651858.CD001134.pub3.www.cochranelibrary.com

Drevets, W. C., Price, J. L., Simpson, J. R., Todd, R. D., Reich, T., Vannier, M., \& Raichle, M. E. (1997). Subgenual prefrontal cortex abnormalities in mood disorders. Nature, 386(6627), 824-827. https://doi.org/10.1038/386824a0

England-Mason, G., Kimber, M., Khoury, J., Atkinson, L., MacMillan, H., \& Gonzalez, A. (2017). Difficulties with emotion regulation moderate the association between childhood history of maltreatment and cortisol reactivity to psychosocial challenge in postpartum women. Hormones and Behavior, 95(March), 44-56. https://doi.org/10.1016/j.yhbeh.2017.07.007

Etkin, A., Egner, T., Peraza, D. M., Kandel, E. R., \& Hirsch, J. (2006). Resolving emotional conflict: a role for the rostral anterior cingulate cortex in modulating activity in the amygdala. Neuron, 51(6), 871-882. https://doi.org/10.1016/j.neuron.2006.07.029

Gingnell, M., Bannbers, E., Moes, H., Engman, J., Sylvén, S., Skalkidou, A., ... Sundström-Poromaa, I. (2015). Emotion reactivity is increased 4-6 weeks postpartum in healthy women: a longitudinal fMRI study. PLOS ONE, 10(6), e0128964. https://doi.org/10.1371/journal.pone.0128964

Goodman, L., Corcoran, C., Turner, K., Yuan, N., \& Green, B. (1998). Assessing traumatic event exposure: General issues and preliminary findings for the Stressful Life Events Screening Questionnaire. Journal of Traumatic Stress, 11(3), 521-542.

Gur, R. C., Sara, R., Hagendoorn, M., Marom, O., Hughett, P., Turner, T., ... Gur, R. E. (2002). A method for obtaining 3-dimensional facial expressions and its standardization for use in neurocognitive studies. Journal of Neuroscience Methods, 115, 137-143.

Hamilton, J. P., Farmer, M., Fogelman, P., \& Gotlib, I. H. (2015). Depressive rumination, the defaultmode network, and the dark matter of clinical neuroscience. Biological Psychiatry, 78(4), 224230. https://doi.org/10.1016/j.biopsych.2015.02.020

Hänsel, A., \& Von Känel, R. (2013). Unconscious fearful priming followed by a psychosocial stress test results in higher cortisol levels. Stress and Health, 29(4), 317-323.

https://doi.org/10.1002/smi.2469

Hellhammer, D. H., Wüst, S., \& Kudielka, B. M. (2009). Salivary cortisol as a biomarker in stress research. Psychoneuroendocrinology, 34(2), 163-171. https://doi.org/10.1016/j.psyneuen.2008.10.026

Henry, J. F., \& Sherwin, B. B. (2012). Hormones and cognitive functioning during late pregnancy and postpartum: a longitudinal study. Behavioral Neuroscience, 126(1), 73-85. https://doi.org/10.1037/a0025540; 10.1037/a0025540

Joormann, J., \& Gotlib, I. H. (2010). Emotion regulation in depression: Relation to cognitive inhibition. Cognition \& Emotion, 24(2), 281-298. https://doi.org/10.1080/02699930903407948 
Kerns, J. G., Cohen, J. D., MacDonald, A. W., Cho, R. Y., Stenger, V. A., \& Carter, C. S. (2004). Anterior cingulate conflict monitoring and adjustments in control. Science, 303(5660), 1023-1026. https://doi.org/10.1126/science.1089910

Kohn, N., Eickhoff, S. B., Scheller, M., Laird, A. R., Fox, P. T., Habel, U., ... Antonio, S. (2014). Neural network of cognitive emotion regulation - an ALE meta-analysis and MACM analysis. Neurolmage, 87, 345-355. https://doi.org/10.1016/j.neuroimage.2013.11.001.Neural

Kudielka, B. M., Hellhammer, D. H., \& Wüst, S. (2009). Why do we respond so differently? Reviewing determinants of human salivary cortisol responses to challenge. Psychoneuroendocrinology, 34(1), 2-18. https://doi.org/10.1016/j.psyneuen.2008.10.004

Laurent, H. K., \& Ablow, J. C. (2012). A cry in the dark: depressed mothers show reduced neural activation to their own infant's cry. Social Cognitive and Affective Neuroscience, 7(2), 125-34. https://doi.org/10.1093/scan/nsq091

Laurent, H. K., \& Ablow, J. C. (2013). A face a mother could love: Depression-related maternal neural responses to infant emotion faces. Social Neuroscience, 8(3), 228-239. https://doi.org/10.1080/17470919.2012.762039

Lucassen, P. J., Pruessner, J., Sousa, N., Almeida, O. F. X., Van Dam, A. M., Rajkowska, G., ... Czéh, B. (2014). Neuropathology of stress. Acta Neuropathologica, 127(1), 109-135. https://doi.org/10.1007/s00401-013-1223-5

Mastorakos, G., \& Ilias, I. (2006). Maternal hypothalamic-pituitary-adrenal axis in pregnancy and the postpartum period: Postpartum-related disorders. Annals of the New York Academy of Sciences, 900(1), 95-106. https://doi.org/10.1111/j.1749-6632.2000.tb06220.x

Mathews, A., \& MacLeod, C. (1984). Cognitive approaches to emotion and emotional disorders. Annual Review of Psychology, 45, 25-50.

Meyer, J. S., \& Novak, M. A. (2012). Minireview: Hair Cortisol: A Novel Biomarker of HypothalamicPituitary-Adrenocortical Activity. Endocrinology, 153(9), 4120-4127. https://doi.org/10.1210/en.2012-1226

O'Hara, M. W., \& McCabe, J. E. (2013). Postpartum depression: Current status and future directions. Annual Review of Clinical Psychology, 9(1), 379-407. https://doi.org/10.1146/annurev-clinpsy050212-185612

Pariante, C. M., \& Lightman, S. L. (2008). The HPA axis in major depression: classical theories and new developments. Trends in Neurosciences, 31(9), 464-468. https://doi.org/10.1016/J.TINS.2008.06.006

Price, J. L., \& Drevets, W. C. (2012). Neural circuits underlying the pathophysiology of mood disorders. Trends in Cognitive Sciences, 16(1), 61-71. https://doi.org/10.1016/j.tics.2011.12.011

Pruessner, J. C., Dedovic, K., Khalili-Mahani, N., Engert, V., Pruessner, M., Buss, C., ... Lupien, S. (2008). Deactivation of the limbic system during acute psychosocial stress: Evidence from positron emission tomography and functional magnetic resonance imaging studies. Biological Psychiatry, 63(2), 234-240. https://doi.org/10.1016/j.biopsych.2007.04.041

Quinete, N., Bertram, J., Reska, M., Lang, J., \& Kraus, T. (2015). Highly selective and automated online SPE LC-MS3 method for determination of cortisol and cortisone in human hair as biomarker for stress related diseases. Talanta, 134, 310-316. https://doi.org/10.1016/j.talanta.2014.11.034

Rigucci, S., Serafini, G., Pompili, M., Kotzalidis, G. D., \& Tatarelli, R. (2010). Anatomical and functional 
correlates in major depressive disorder: the contribution of neuroimaging studies. The World Journal of Biological Psychiatry, 11(2 Pt 2), 165-180.

https://doi.org/10.1080/15622970903131571

Roberts, A. C., \& Wallis, J. D. (2000). Inhibitory control and affective processing in the prefrontal cortex: neuropsychological studies in the common marmoset. Cerebral Cortex, 10(3), 252-262. https://doi.org/10.1093/cercor/10.3.252

Roelofs, K., Bakvis, P., Hermans, E. J., van Pelt, J., \& van Honk, J. (2007). The effects of social stress and cortisol responses on the preconscious selective attention to social threat. Biological Psychology, 75(1), 1-7. https://doi.org/10.1016/j.biopsycho.2006.09.002

Roelofs, K., Elzinga, B. M., \& Rotteveel, M. (2005). The effects of stress-induced cortisol responses on approach-avoidance behavior. Psychoneuroendocrinology, 30(7), 665-677. https://doi.org/10.1016/j.psyneuen.2005.02.008

Scharlau, F., Pietzner, D., Vogel, M., Gaudl, A., Ceglarek, U., Thiery, J., ... Kiess, W. (2017). Evaluation of hair cortisol and cortisone change during pregnancy and the association with self-reported depression, somatization, and stress symptoms. Stress, 1-8. https://doi.org/10.1080/10253890.2017.1392507

Schlotz, W., Kumsta, R., Layes, I., Entringer, S., Jones, A., \& Wüst, S. (2008). Covariance between psychological and endocrine responses to pharmacological challenge and psychosocial stress: A question of timing. Psychosomatic Medicine, 70(7), 787-796. https://doi.org/10.1097/PSY.0b013e3181810658

Seth, S., Lewis, A. J., \& Galbally, M. (2016). Perinatal maternal depression and cortisol function in pregnancy and the postpartum period: a systematic literature review. BMC Pregnancy and Childbirth, 16(1), 124. https://doi.org/10.1186/s12884-016-0915-y

Shackman, A. J., Salomons, T. V, Slagter, H. A., Andrew, S., Winter, J. J., \& Davidson, R. J. (2011). The integration of negative affect, pain, and cognitive control in the cingulate cortex. Nature Reviews Neuroscience, 12(3), 154-167. https://doi.org/10.1038/nrn2994.The

Smith, I. D., \& Shearman, R. P. (1974). Fetal plasma steroids in relation to parturition. I. The effect of gestational age upon umbilical plasma corticosteroid levels following vaginal delivery. BJOG: An International Journal of Obstetrics and Gynaecology, 81(1), 11-15. https://doi.org/10.1111/j.1471-0528.1974.tb00357.x

Smith, R., Mesiano, S., \& McGrath, S. (2002). Hormone trajectories leading to human birth. Regulatory Peptides, 108(2-3), 159-164. https://doi.org/10.1016/S0167-0115(02)00105-2

Spielberg, J. M., Stewart, J. L., Levin, R. L., Miller, G. A., \& Heller, W. (2008). Prefrontal Cortex, Emotion, and Approach/Withdrawal Motivation. Social and Personality Psychology Compass, 2(1), 135-153. https://doi.org/10.1111/j.1751-9004.2007.00064.x

Stalder, T., Steudte, S., Miller, R., Skoluda, N., Dettenborn, L., \& Kirschbaum, C. (2012). Intraindividual stability of hair cortisol concentrations. Psychoneuroendocrinology, 37(5), 602-610. https://doi.org/10.1016/j.psyneuen.2011.08.007

Stuss, D. T., \& Alexander, M. P. (2007). Is there a dysexecutive syndrome? Philosophical Transactions of the Royal Society B: Biological Sciences, 362(1481), 901-915. https://doi.org/10.1098/rstb.2007.2096

Sudheimer, K. D., Abelson, J. L., Taylor, S. F., Martis, B., Welsh, R. C., Warner, C., ... Liberzon, I. (2013). Exogenous glucocorticoids decrease subgenual cingulate activity evoked by sadness. 
Neuropsychopharmacology, 38(5), 826-845. https://doi.org/10.1038/npp.2012.249

Sutton, S. K., \& Davidson, R. J. (1997). Prefrontal brain asymmetry: A Biological Substrate of the Behavioral Approach and Inhibition Systems. Psychological Science, 8(3), 204-210. https://doi.org/10.1111/j.1467-9280.1997.tb00413.x

van Honk, J., Tuiten, A., van den Hout, M., Koppeschaar, H., Thijssen, J., de Haan, E., \& Verbaten, R. (2000). Conscious and preconscious selective attention to social threat: different neuroendocrine response patterns. Psychoneuroendocrinology, 25(6), 577-91. https://doi.org/10.1016/S0306-4530(00)00011-1

Varghese, F. P., \& Brown, E. S. (2001). The hypothalamic-pituitary-adrenal axis in major depressive disorder: A brief primer for primary care physicians. Primary Care Companion to the Journal of Clinical Psychiatry, 3(4), 151-155. Retrieved from http://www.ncbi.nlm.nih.gov/pubmed/15014598

Wennig, R. (2000). Potential problems with the interpretation of hair analysis results. Forensic Science International, 107(1-3), 5-12. https://doi.org/10.1016/S0379-0738(99)00146-2

Williams, J. M., Mathews, A., \& MacLeod, C. (1996). The emotional Stroop task and psychopathology. Psychological Bulletin, 120(1), 3-24. Retrieved from http://www.ncbi.nlm.nih.gov/pubmed/8711015 